\title{
Fast MHD oscillations in line-tied homogeneous coronal loops ${ }^{\star}$
}

\author{
A. J. Díaz ${ }^{1}$, R. Oliver ${ }^{1}$, J. L. Ballester ${ }^{1}$, and B. Roberts ${ }^{2}$ \\ 1 Departament de Física, Universitat de les Illes Balears, 07122 Palma de Mallorca, Spain \\ e-mail: toni@hubble.uib.es; [ramon.oliver;dfsjlbo]@uib.es \\ 2 School of Mathematics and Statistics, University of St. Andrews, St. Andrews, Fife KY16 9SS, Scotland, UK \\ e-mail: bernie@mcs.st-andrews.ac.uk
}

Received 18 November 2003 / Accepted 3 June 2004

\begin{abstract}
Loop oscillations have been abundantly reported in recent years. Earlier analytical studies of loop oscillations consider freely propagating waves, allowing for line-tying by a quantization of the wavenumber. Here we consider the rich spectrum of fast MHD modes (both standing and leaky) in coronal loops, allowing for line-tying and performing some comparisons with observational data. We point out that in a straight and homogeneous cylindrical flux tube there should be observational signatures of the excitation of higher order harmonics. Our results indicate that these modes become leaky with the addition of the chromospheric structure at the base of the loop. Leakage can be quite efficient in damping the oscillations for many of these high frequency (compared to fundamental) modes.
\end{abstract}

Key words. Sun: oscillations - Sun: magnetic fields - Sun: corona - Sun: prominences

\section{Introduction}

It is clear from $\mathrm{X}$-rays observations that the solar corona is structured in regions with open magnetic fields, or coronal holes, and regions with closed magnetic field (with both endpoints of magnetic field lines anchored in the photosphere), with small-scale energetic features ( $X$-ray bright points) scattered over all the Sun's surface. Closed regions are in fact composed of myriads of coronal loops, with a wide range of densities and temperatures. Coronal loop oscillations have been widely reported, specially using data from the Transition Region and Coronal Explorer (TRACE) and the SUMER instrument of the Solar and Heliospheric Observatory (SOHO). Specifically, there is evidence of standing oscillations in coronal loops (Aschwanden et al. 1999, 2002; Nakariakov et al. 1999; Schrijver \& Brown 2000; Schrijver et al. 2002; Ofman \& Wang 2002; Wang et al. 2002, 2003a,b). These loop oscillations have recently been reviewed by Aschwanden (2002) and Roberts (2000, 2002).

Coronal loops are mainly modelled as magnetic flux tubes, perhaps with twist. However, to study their oscillatory properties the models tend to be quite simple, with the aim of studying the most relevant and generic features first and then adding other effects. The most simple model, the one used in this paper, is a cylindrical flux tube in isothermal equilibrium, without gravity, curvature, radiation or extra terms of heating. This leads to a profile with constant pressure along the tube

* Appendix A is only available in electronic form at http://www. edpsciences.org (see, for example, Priest et al. 2000, for a discussion of different equilibrium profiles).

Here we consider the effect of adding a photospheric linetying boundary condition: the photospheric plasma is so dense that perturbations generated in the corona must vanish on the boundary. Our analysis is an extension of the basic case of an infinite homogeneous flux tube (Edwin \& Roberts 1983). There are many other effects that can shift the frequency or change the oscillatory properties, although they are usually studied under other simplifying assumptions or with numerical methods: curvature (Smith et al. 1997), a twist in the equilibrium magnetic field (Bennett et al. 1999; Sakai et al. 2000), stratification in a slender flux tube (Hollweg \& Roberts 1981), flows in an infinite flux tube (Nakariakov \& Roberts 1995; Somasundaram et al. 1999; Homem et al. 2003), non-ideal MHD effects such as resonant absorption (Steinolfson \& Davila 1993; Ofman et al. 1994, 1995; Ruderman \& Roberts 2002; Goossens et al. 2002) or phase mixing (Heyvaerts \& Priest 1983), non-stationary response to prescribed perturbations (Goedbloed 1983; Poedts \& Goossens 1991). In fact, a full theory of coronal loop oscillations should take into account all of these features, but for the sake of simplicity they are neglected here.

It is of interest to investigate a more simple model, because the modes of an infinite flux tube have proved useful in explaining many observations (e.g., Roberts et al. 1984; Nakariakov et al. 1999; Nakariakov \& Ofman 2001) or as a starting point for the inclusion of other effects (e.g., Ruderman \& Roberts 2002; Goossens et al. 2002). The effect of a chromospheric layer on the Alfvén modes was studied in one dimension by 


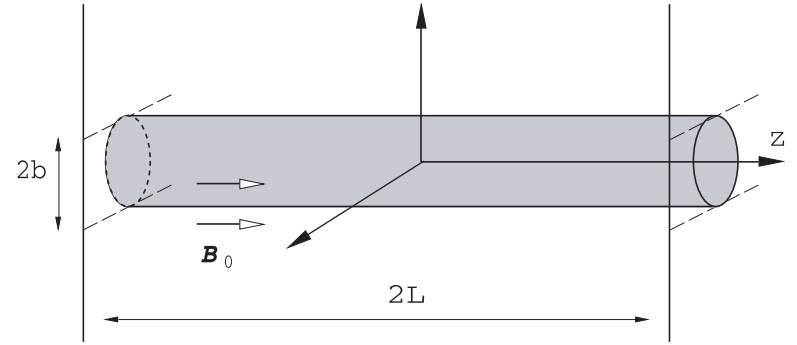

Fig. 1. Sketch of the equilibrium configuration of a homogeneous flux tube, in which the shaded grey zone represents the cold and dense plasma of the loop. The density inside the loop is $\rho_{1}$ and in the coronal environment $\rho_{\mathrm{c}}$, while the tube footpoints are located in the photosphere (and subject to line-tying) at the positions $z= \pm L$, so its length is $2 L$. The magnetic field is uniform and parallel to the $z$-axis, and the whole configuration is invariant in the azimuthal direction.

Hollweg (1984), De Pontieu et al. (2001) and Ofman (2002), focusing on the calculation of the reflection and transmission coefficients and the damping due to non-ideal effects. Here we use ideal MHD and consider the effects produced by the addition of line-tying and the inclusion of a chromospheric layer on the fast modes of oscillation of a cylindrical flux tube. In this case, the only possibility for damping is leakage to the coronal medium, such as was studied in Cally (2003) for and unbounded homogeneous flux tube.

This paper is organized as follows. In Sect. 2 the dispersion relations for cylindrical geometry are presented and the special case of a thin flux tube is studied. In Sect. 3 the non-leaky spectrum is presented, while in Sect. 4 the leaky modes are considered and some estimates of the results for realistic parameters are computed. In Sect. 5, the effect of adding a chromosphere at the base of the loop is studied. Finally in Sect. 6 our conclusions are presented. In the appendix, we study the line-tied fast modes in a Cartesian slab, which provides a useful comparison with the results for cylindrical geometry.

\section{Dispersion relations for line-tied cylindrical homogeneous flux tubes}

\subsection{Equilibrium model and basic equations}

The equilibrium configuration consists of a single homogeneous straight flux tube of length $2 L$ embedded in a magnetic environment (Fig. 1). The magnetic field is uniform and parallel to the $z$-axis $\left(\boldsymbol{B}_{0}=B_{0} \hat{z}\right)$ and the plasma density inside the loop is $\rho_{\mathrm{l}}$, while it is $\rho_{\mathrm{c}}$ in the surrounding coronal medium. The radius of the tube is $b$. Notice that in this simple model the temperature and pressure are assumed to be uniform (for a more detailed discussion about the equilibrium profile of coronal loops see Priest et al. 2000; Aschwanden \& Schrijver 2002; and Testa et al. 2002).

In the study of the oscillations of the present structure we restrict ourselves to perturbations in a zero-beta plasma, in which the slow mode is absent $\left(v_{z}=0\right)$. The following partial differential equations are derived for the total pressure perturbation and the remaining velocity components (Roberts 1991; Díaz et al. 2002),

$\left(\frac{\partial^{2}}{\partial t^{2}}-c_{\mathrm{A}}^{2} \nabla^{2}\right) p_{\mathrm{T}}=0$,

$\rho_{0}\left(\frac{\partial^{2}}{\partial t^{2}}-c_{\mathrm{A}}^{2} \frac{\partial^{2}}{\partial z^{2}}\right) \boldsymbol{v}_{\perp}+\nabla_{\perp} \frac{\partial p_{\mathrm{T}}}{\partial t}=0$,

where the symbol $\perp$ stands for the components perpendicular to $\boldsymbol{B}_{0}$. Notice that $p_{\mathrm{T}}=0$ satisfies Eq. (1); this solution corresponds to Alfvén modes. Solutions with $p_{\mathrm{T}} \neq 0$ are obtained from Eqs. (1) and (2); these solutions are the fast modes.

\subsection{Analytical solution}

Since we are looking for stationary states, the time component is Fourier-analyzed in the form $\mathrm{e}^{-\mathrm{i} \omega t}$, and our aim is to calculate the set of allowed frequencies for the model. We use cylindrical geometry, since it seems more suitable to model coronal loops, although some expressions are also derived for a plasma slab modelled by a Cartesian coordinate system (see the appendix). The standard method for solving this kind of problem in a bounded region is separation of variables of the form

$p_{\mathrm{T}}(r, \varphi, z)=u(r) \Phi(\varphi) h(z)$,

$\left(\frac{\mathrm{d}^{2}}{\mathrm{~d} z^{2}}+\frac{\omega^{2}}{c_{\mathrm{A}}^{2}}\right) h(z)=-\lambda^{2} h(z)$,

$\frac{\mathrm{d}^{2} \Phi(\varphi)}{\mathrm{d} \varphi^{2}}=-m^{2} \Phi(\varphi) \Rightarrow \Phi(\varphi)=\mathrm{e}^{\mathrm{i} m \varphi}, m=0,1,2, \ldots$,

$\frac{1}{r} \frac{\mathrm{d}}{\mathrm{d} r}\left(r \frac{\mathrm{d} u(r)}{\mathrm{d} r}\right)-\left(\lambda^{2}+\frac{m^{2}}{r^{2}}\right) u(r)=0$.

Now, if there is no equilibrium inhomogeneity, from Eq. (4) it immediately follows that the $z$-dependent part of $p_{\mathrm{T}}$ is in the form of trigonometric functions, namely

$h(z)=D \sin \kappa(z+L)$.

The wavenumber $\kappa$ is defined as

$\kappa^{2}=\lambda^{2}+\frac{\omega^{2}}{c_{\mathrm{A}}^{2}}$.

It is necessary to write the perturbed velocity components in terms of the perturbed pressure by using Eq. (2),

$\boldsymbol{v}_{\perp}=\frac{\mathrm{i} \omega}{\rho_{0} c_{\mathrm{A}}^{2}} \frac{1}{\lambda^{2}} \nabla_{\perp} p_{\mathrm{T}}$.

The photospheric line-tying boundary condition that $\boldsymbol{v}_{\perp}=0$ on the surfaces $z= \pm L$ leads, from Eq. (9), to the condition

$p_{\mathrm{T}}(z= \pm L)=0$,

which results in the quantization of $\kappa$ in the form

$\kappa=\frac{(n+1) \pi}{2 L}, \quad n=0,1,2, \ldots$, 
for even or odd modes about $z=0$ when the number $n$ is even or odd, respectively. Consequently, the $\kappa$ and $\lambda$ in Eq. (8) should be labelled by the integer $n=0,1,2, \ldots$, although we omit this for the sake of clarity. The normalization constant $D$ in Eq. (7) is then chosen to fulfil the orthonormality of the functions $h(z)$,

$D=\frac{1}{\sqrt{L}}$

It is important to remark that Eq. (4) together with the linetying condition leads to a Stürm-Liouville problem, so the solutions found are a complete basis, and therefore any arbitrary solution of Eq. (1) can be expressed as a sum of these functions.

It is also necessary to use "jump" conditions to connect the solution in the interior and the exterior regions. On a boundary on which $\boldsymbol{n} \cdot \boldsymbol{B}=0$ is satisfied, where $\boldsymbol{n}$ is the vector normal to the boundary, these conditions are (Goedbloed 1983)

$\boldsymbol{n} \cdot[\boldsymbol{v}]=0, \boldsymbol{n} \cdot[\boldsymbol{B}]=0, \quad\left[p_{\mathrm{T}}\right]=0$.

Next, we deal with the radial part of the solution, for which it is more convenient to work with $\lambda^{*}=i \lambda$ in Eqs. (4) and (6). Equation (6) is the modified Bessel differential equation, so its general solution can be written as a combination of Bessel functions. In the coronal region, hereafter labelled (c), the suitable solution is a Hankel function of first kind because of the exponential decay for long values of the argument, while in the loop region, labelled (1), it must be a Bessel function $J_{m}$ to satisfy the requirement of regularity at the loop axis. Then,

$u(r)= \begin{cases}A J_{m}\left(\lambda^{(1)^{*}} r\right), & 0 \leq r \leq b, \\ B H_{m}^{(1)}\left(\lambda^{(\mathrm{c})^{*}} r\right), & r>b .\end{cases}$

Now, from Eq. (9) the spatial dependence of $v_{r}$ is given by

$v_{r}(r, \phi, z)=\frac{\mathrm{i} \omega}{\rho_{0} c_{\mathrm{A}}} \begin{cases}A J_{m}{ }^{\prime}\left(\lambda^{(1)^{*}} r\right) / \lambda^{(1)^{*}}, & 0 \leq r \leq b, \\ B H_{m}^{(1)^{\prime}}\left(\lambda^{(\mathrm{c})^{*}} r\right) / \lambda^{(\mathrm{c})^{*}}, & r>b,\end{cases}$

where the dash denotes the derivative of a function with respect to its argument. Here it should be remarked that the product $\rho_{0} c_{\mathrm{A}}^{2}=B_{0}^{2} / \mu$ has the same value in all the regions. Imposing the boundary conditions Eq. (13) on the surface $r=b$ leads to the dispersion relation

$\frac{1}{\lambda^{(\mathrm{c})^{*}}} \frac{H_{m}^{(1)^{\prime}}\left(\lambda^{(\mathrm{c})^{*}} b\right)}{H_{m}^{(1)}\left(\lambda^{(\mathrm{c})^{*}} b\right)}-\frac{1}{\lambda^{(1)^{*}}} \frac{J_{m}{ }^{\prime}\left(\lambda^{(1)^{*}} b\right)}{J_{m}\left(\lambda^{(1)^{*}} b\right)}=0$.

This is a particular form of other well-known dispersion relations (Edwin \& Roberts 1983; Cally 1986; Karami et al. 2002).

Notice that in the dispersion relation (and also in Eqs. (A.3) and (A.4) for a slab, discussed in the appendix) the external wavenumber $\lambda^{(\mathrm{c})}$ can be real or imaginary, thus we are not restricted to trapped modes. Also notice that a so called surface mode is obtained if $\lambda^{(1)}$ is real, and a body mode if $\lambda^{(1)^{*}}=i \lambda^{(1)}$ is purely imaginary. However, in the infinite homogeneous tube there are no trapped surface modes for a low-beta plasma.

In the following discussion, magnitudes will be nondimensionalized against $L$, the half-length of the magnetic flux tube, and $c_{\mathrm{Ac}}$, the Alfvén speed in the coronal environment.

\subsubsection{Trapped modes in the $b / L \rightarrow 0$ limit}

This special case is interesting mainly because in the solar atmosphere structures tend to be thin in comparison with the length of the supporting magnetic field lines. It is similar to the limit $\kappa a \rightarrow 0$ in Edwin \& Roberts (1983), taking into account that $L \sim 1 / \kappa$.

For trapped modes it is necessary that $\lambda^{*}$ be a purely imaginary number, so the external solution can be expressed in terms of the modified Bessel function $K_{m}$, and under this condition the dispersion relation, Eq. (16), can be rewritten as (Edwin \& Roberts 1983)

$\frac{1}{\lambda^{(\mathrm{c})}} \frac{K_{m}{ }^{\prime}\left(\lambda^{(\mathrm{c})} b\right)}{K_{m}\left(\lambda^{(\mathrm{c})} b\right)}-\frac{1}{\lambda^{(1)}} \frac{I_{m}{ }^{\prime}\left(\lambda^{(1)} b\right)}{I_{m}\left(\lambda^{(1)} b\right)}=0$.

Notice that, since $\lambda^{(\mathrm{c})}$ and $\lambda^{(\mathrm{l})}$ remain finite in the limit $b / L \rightarrow 0$, the arguments of the Bessel functions in this expression also tend to zero in this limit.

Now it is necessary to invoke series expansions for the Bessel functions and their derivatives for $|z| \ll 1$ (Abramowitz $\&$ Stegun 1967). There are two possibilities, $m=0$ and $m \neq 0$. If $m=0$, then $K_{0}\left(\lambda^{(\mathrm{c})} b\right) \sim \log \lambda^{(\mathrm{c})} b$ and there is no possibility of balancing the two terms in Eq. (17), so no sausage $(m=0)$ solution exists in the thin tube limit. If $m \neq 0$, then Eq. (17) can be written (after some algebra) as

$\lambda^{(\mathrm{c})^{2}}+\lambda^{(1)^{2}}=0$

which leads to

$\frac{\omega}{c_{\mathrm{Ac}}} L=\frac{c_{\mathrm{k}}}{c_{\mathrm{Ac}}} \kappa L=\sqrt{\frac{2}{1+\rho_{\mathrm{l}} / \rho_{\mathrm{c}}}} \frac{(n+1) \pi}{2}$.

Here $c_{\mathrm{k}}$ denotes the kink speed, an intermediate speed between the lower Alfvén speed of the tube and the higher one of the surrounding coronal medium,

$c_{\mathrm{k}}=\sqrt{\frac{\rho_{\mathrm{l}} c_{\mathrm{Al}}^{2}+\rho_{\mathrm{c}} c_{\mathrm{Ac}}^{2}}{\rho_{\mathrm{l}}+\rho_{\mathrm{c}}}}=c_{\mathrm{Ac}} \sqrt{\frac{2}{1+\rho_{\mathrm{l}} / \rho_{\mathrm{c}}}}$.

The equalities in this expression come from our assumption of equal magnetic field inside and outside the tube. It is worth noticing that the kink $(m=1)$ and all the fluting $(m \geq 2)$ modes tend to the same value of the frequency in the limit $b / L \rightarrow 0$.

From this development an important difference between the kink mode in a slab and a tube becomes evident: for a thin slab $\lambda^{\text {(c) }} \rightarrow 0$ (from Eq. (A.6)), but in a tube with $b / L \rightarrow 0$ Eqs. (8) and (19) give

$\lambda^{(\mathrm{c})^{2}}=\kappa^{2}\left(1-\frac{c_{\mathrm{k}}^{2}}{c_{\mathrm{Ac}}^{2}}\right)$,

so that $\lambda^{(\mathrm{c})}$ is different from 0 . The immediate consequence of this different behaviour is that in a slab the perturbation reaches long distances from the slab axis (see the appendix), while in a cylinder it remains confined for arbitrarily small values of the radius. For thick structures the results of cylindrical loops and Cartesian slabs are in a good agreement (e.g., Díaz et al. 2002), with only minor shifts in frequency but similar spatial structure along and across the magnetic field. 


\section{Trapped modes of line-tied coronal tubes}

The next step is to study the solutions of Eq. (16), focusing on the set of frequencies of trapped modes. It is important to remark that $\lambda^{(\mathrm{c})}$ has to be real to have trapped modes, requiring

$$
\frac{\omega}{c_{\mathrm{Ac}}} \leq \kappa \text {. }
$$

Therefore, there is a cut-off frequency, $\omega_{\text {cut }}$, for each value of $\kappa$,

$$
\frac{\omega_{\mathrm{cut}}}{c_{\mathrm{Ac}}} L=\frac{(n+1) \pi}{2}
$$

In Fig. 2 the variation of the mode frequencies with the tube radius is plotted for a high density flux tube, while in Fig. 3 a density ratio typical of coronal loops has been selected. In the less dense tube there are fewer trapped modes, but otherwise the structure of the dispersion diagrams is quite similar. Each mode can be labelled by three numbers: $n$ for the $z$-wavenumber in Eq. (11), related to the number of extrema in the $z$-direction; $m$ for the azimuthal wavenumber in Eq. (5) and in the Bessel functions in Eq. (14), representing the sausage ( $m=0)$, kink $(m=1)$ and fluting $(m \geq 2)$ modes; and another one (say $l$ ) for the number of extrema in the $r$-direction. Each family of modes having the same value of $n$ have a cut-off frequency given by Eq. (23), which modes belonging to other families can cross.

In fact, Figs. 2 and 3 are just the low-beta limit $\left(c_{\mathrm{s}} \rightarrow 0\right)$ of Fig. 4 in Edwin \& Roberts (1983), although in that figure the fluting modes are not depicted. However, it should be taken into account that their plot is drawn using the dependent variable $\omega / \kappa$, while in Figs. 2 and $3 \omega L / c_{\text {Ac }}$ is used, so each family of modes has a different vertical (and also horizontal) scale.

Finally, in the thin tube limit, it is easy to check that Eq. (19) (valid for $b / L \rightarrow 0$ ) is satisfied, so for each family of modes with the same $n$ the sausage mode $(m=0)$ is no longer trapped and its frequency is above the cut-off, while the kink and all fluting modes tend to $(n+1) \pi / 2 c_{\mathrm{k}} / c_{\mathrm{Ac}}=0.157(n+1)$ for $\rho_{\mathrm{l}} / \rho_{\mathrm{c}}=200$ and $0.670(n+1)$ for $\rho_{\mathrm{l}} / \rho_{\mathrm{c}}=10$, in agreement with Figs. 2 and 3, respectively.

\section{Leaky modes of line-tied coronal tubes}

Following our study of the solutions of trapped modes, we next deal with the more general case of leaky modes, allowing the frequency to become complex. We use the notation $\operatorname{Re}\{\omega\}=\omega_{\mathrm{r}}, \operatorname{Im}\{\omega\}=\omega_{\mathrm{i}}, \operatorname{Re}\left\{\lambda^{(\mathrm{c})^{*}}\right\}=\lambda_{\mathrm{r}}^{(\mathrm{c})}$ and $\operatorname{Im}\left\{\lambda^{(\mathrm{c})^{*}}\right\}=\lambda_{\mathrm{i}}^{(\mathrm{c})}$ to denote the real and imaginary parts of $\omega$ and $\lambda^{(\mathrm{c})}$. The starting point is that all solutions of the dispersion relation, Eq. (16), satisfy either

$\lambda_{\mathrm{r}}^{(\mathrm{c})} \geq 0, \quad \lambda_{\mathrm{i}}^{(\mathrm{c})} \leq 0, \quad \omega_{\mathrm{r}} \geq 0, \quad \omega_{\mathrm{i}} \leq 0$

or

$\lambda_{\mathrm{r}}^{(\mathrm{c})} \geq 0, \quad \lambda_{\mathrm{i}}^{(\mathrm{c})} \leq 0, \quad \omega_{\mathrm{r}} \leq 0, \quad \omega_{\mathrm{i}} \geq 0$.

Therefore, we proceed to study the properties of these two types of solutions. The temporal dependence can be written in the following way

$\mathrm{e}^{-\mathrm{i} \omega t}=\mathrm{e}^{-\mathrm{i} \omega_{\mathrm{r}} t} \mathrm{e}^{\omega_{\mathrm{i}} t}=\mathrm{e}^{-\mathrm{i} \omega_{\mathrm{r}} t} \mathrm{e}^{-t / \tau}$,
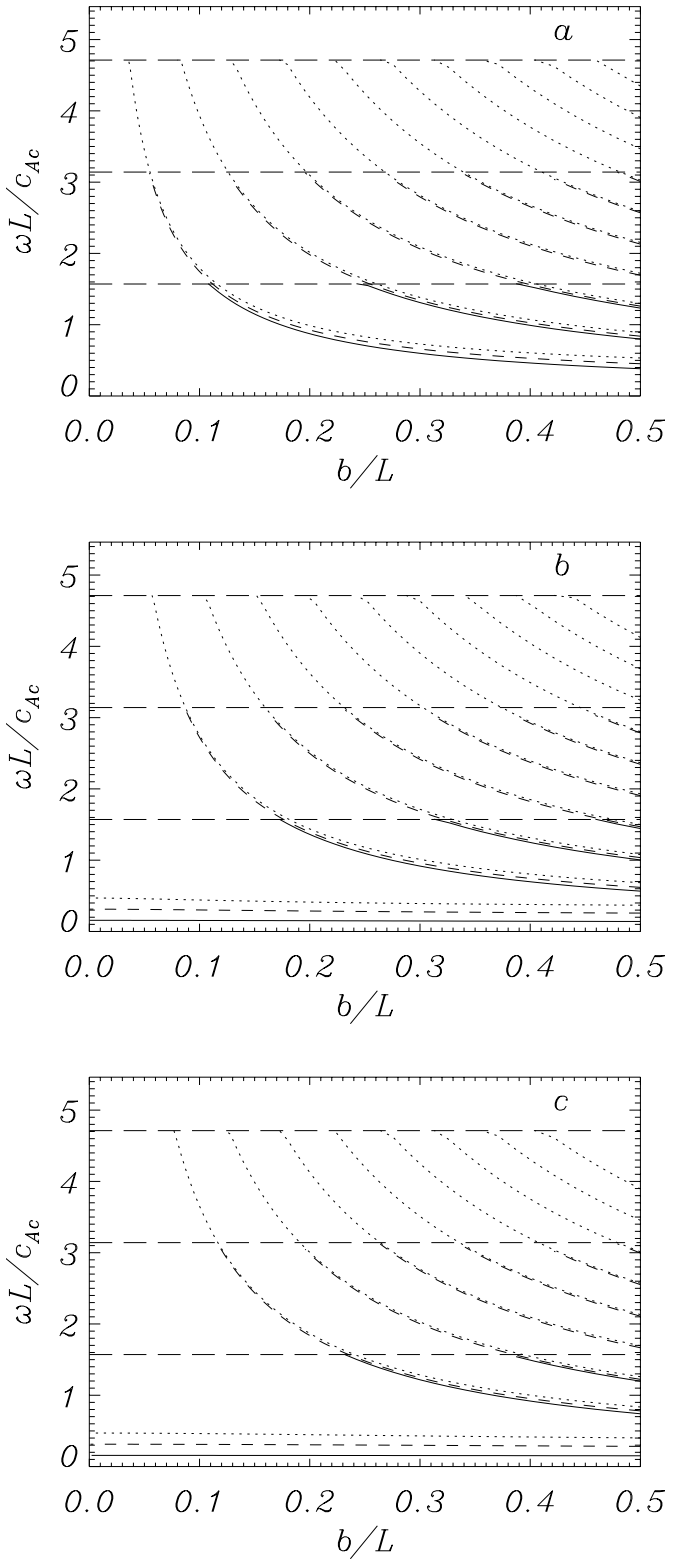

Fig. 2. High density flux tubes: variation of the dimensionless frequency, $\omega L / c_{\mathrm{Ac}}$, with $b / L$, the tube radius as a fraction of loop halflength, for modes $n=0$ (even, solid lines), $n=1$ (odd, dashed lines) and $n=2$ (even, dotted lines) of a tube with density ratio $\rho_{1} / \rho_{\mathrm{c}}=200$. The modes represented are a) sausage modes $(m=0)$, b) kink modes $(m=1)$ and c) first fluting modes $(m=2)$. The cut-off frequencies are marked with horizontal long dashed lines.

with a damping time, $\tau$, defined as

$\tau=-1 / \omega_{\mathrm{i}}$

The next step is to take into account the spatial dependence for large values of $r$. It is convenient to use the expansion of the Hankel function for large arguments, namely $H_{m}^{(1)}(z) \sim \mathrm{e}^{-\mathrm{i} z}$, $|z| \gg 1$ (Abramowitz \& Stegun 1967). Thus,

$p_{\mathrm{T}} \sim \mathrm{e}^{\mathrm{i} \omega t} H_{m}^{(1)}\left(\lambda^{(\mathrm{c})} r\right) \sim \mathrm{e}^{-t / \tau} \mathrm{e}^{\lambda_{\mathrm{i}}^{(\mathrm{c})} r} \mathrm{e}^{-\left(\lambda_{\mathrm{r}}^{(\mathrm{c})} r+\mathrm{i} \omega_{\mathrm{r}} t\right)}$,

when $r \rightarrow \infty$.

Now it is evident that solutions satisfying Eq. (24) correspond to waves propagating away from the density 

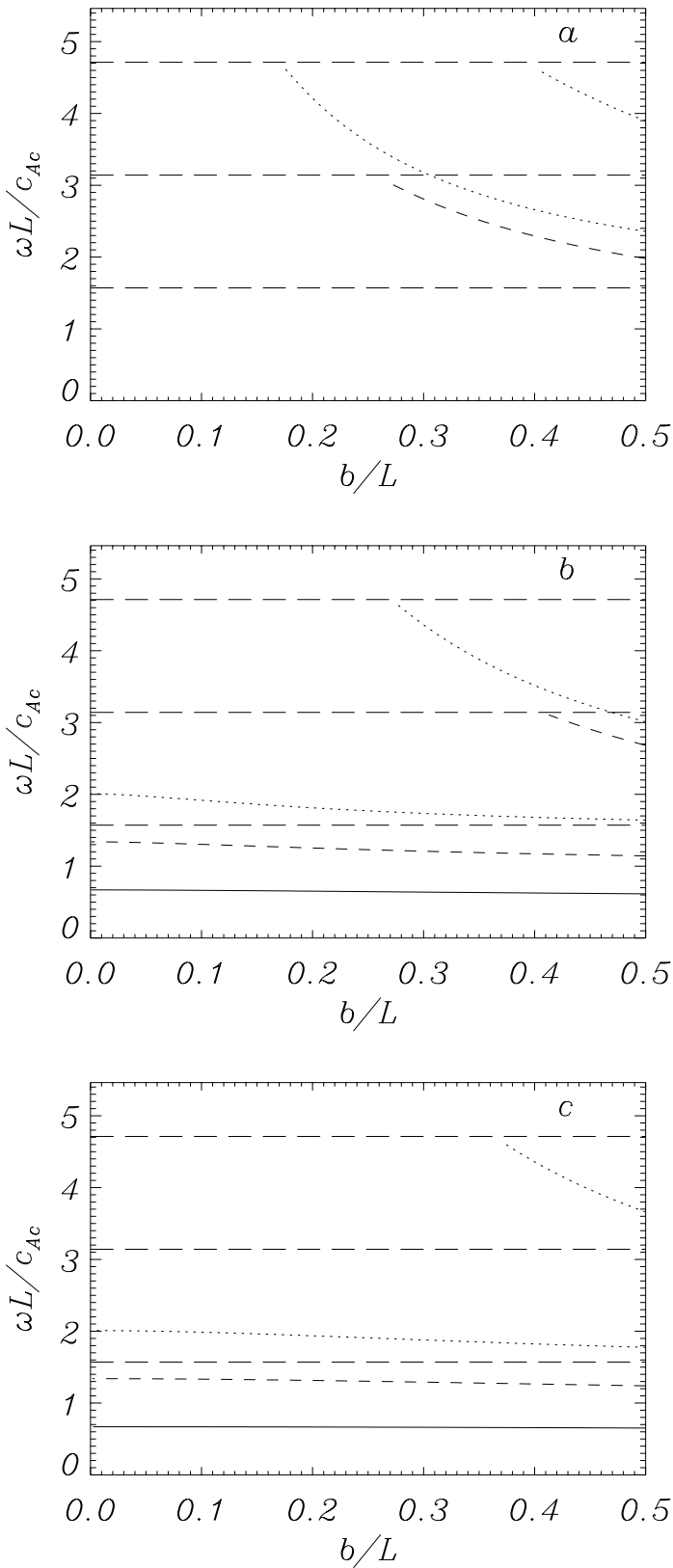

Fig. 3. Coronal loops: variation of the dimensionless frequency with the tube radius for modes $n=0$ (even, solid lines), $n=1$ (odd, dashed lines) and $n=2$ (even, dotted lines) of a tube with density ratio $\rho_{\mathrm{l}} / \rho_{\mathrm{c}}=10$. The modes represented are a) sausage modes $(m=0)$, b) kink modes $(m=1)$ and c) first fluting modes $(m=2)$. The cutoff frequencies are marked with horizontal long dashed lines. Notice that in panel a) the sausage modes with $n=1$ (solid lines) are absent because all of them are leaky.

enhancement and exponentially decaying with time, but growing exponentially for large $r$. On the other hand, the solutions satisfying Eq. (25) correspond to waves decaying exponentially for large $r$, but propagating inwards and, even worse, exponentially increasing with time. Hence, these solutions are discarded. Selecting a time dependence of the form $\mathrm{e}^{\mathrm{i} \omega t}$, the other sign for $\lambda^{\text {(c) }}$ in Eq. (8) or the Hankel function of second order $H_{m}^{(2)}(z)$ do not lead to any different solutions from the ones described here. Also, the addition of both functional dependences in the coronal region instead of only one in Eq. (14) would lead to a scattering problem, which is beyond the scope of this work.

The appearance of a solution that grows exponentially away from the cylinder was already noticed in Cally (1986, 2003) and Stenuit et al. (1998). The interpretation of this fact is that the larger the distance from the tube, the earlier the perturbation departed from the tube and, because of the exponential growth of Eq. (28) for reversed time, the bigger the amplitude at that moment in the density enhancement. This is an unphysical solution, since it is unbounded and not square integrable, but we are looking for stationary states, and a leaky wave can only be stationary with this behaviour; hence for more appropriate modeling to this problem the full temporal dependence should be used instead of a Fourier decomposition. Spatial plots of $p_{\mathrm{T}}(r)$ are shown in Fig. 4 for a parameter set typical of coronal loops; it is clear that functions grow exponentially far away from the tube. It is interesting to remark that near the tube there is a region where the amplitude decreases and so the solution seems to be exponentially decreasing, but for larger $r$ it diverges.

Now the leaky modes of a cylindrical flux tube, which were discussed admirably by Cally (1986) without line-tying boundary conditions, can be studied from the dispersion relations of Eq. (16). The resulting plots of the remaining fast modes are shown in Fig. 5 for the $n=0$ sausage, kink and first fluting modes; note that the trapped modes with $n=0$ of Fig. 3 (solid lines there) are shown alongside their respective leaky modes. It is clear that when the frequency of a trapped mode surpasses the corresponding cut-off, the mode becomes leaky, but otherwise its properties are not changed (for example, the number of extrema of $p_{\mathrm{T}}$ in the tube).

Now we consider parameter values appropriate for coronal loops. In Nakariakov et al. (1999) a tube with length $2 L=$ $130 \mathrm{Mm}$, diameter $2 b=1 \mathrm{Mm}$ was observed, a density ratio $\rho_{\mathrm{l}} / \rho_{\mathrm{c}} \approx 10$ was assumed and the resulting observed period was $T=4.27$ min with an exponential decay with a damping time $\tau=14.5 \mathrm{~min}$. This gives the value $b / L=0.015$; using the coronal particle number $n_{\mathrm{p}}=5 \times 10^{14} \mathrm{~m}^{-3}$ and a magnetic field strength $B_{0}=10 \mathrm{G}$, we derive the coronal Alfvén speed $c_{\mathrm{Ac}}=2.8 \times 10^{12} B_{0} n_{\mathrm{p}}^{-1 / 2}=1252 \mathrm{~km} \mathrm{~s}^{-1}$. The resulting frequencies, periods and damping times of leaky modes are presented in Table 1 . The higher leaky modes have a damping time of the same order, but a much higher frequency (a lower period). Notice that these modes have periods that are too short to be detected by TRACE (although they may have been reported in eclipse measurements by Katsiyannis et al. 2003) and are also rapidly attenuated by leakage. For trapped modes with higher order $n$, the periods are those given in Table 1 divided by $(n+1)$ (see Eq. (19)), so modes with shorter wavelength along the loop have smaller periods than those shown in this table. On the other hand, the real and imaginary parts of the frequency of leaky modes for $n \geq 1$ take very similar values for fixed $m$ and $l$. This is apparent in Fig. 2, in which the frequencies of modes with different values of $n$ almost overlap, a trend found also for the real part of the frequencies of the leaky modes (Fig. 5). 

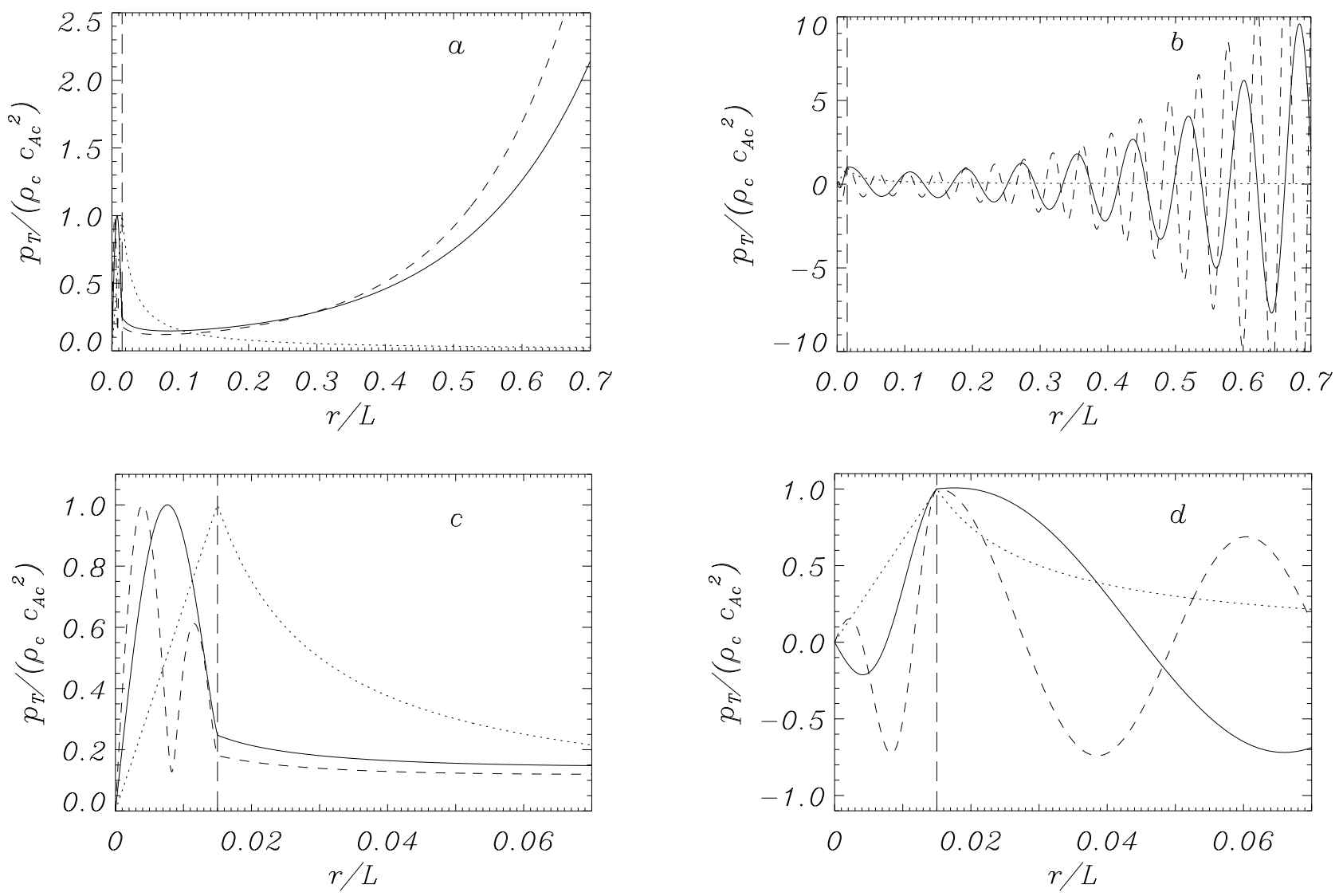

Fig. 4. Cuts in the direction $z=0$ of $\mathbf{a})$ the modulus and $\mathbf{b})$ the imaginary part of $p_{\mathrm{T}}$ for the kink even modes $(n=0, m=1)$. The fundamental mode $(l=1)$, which is trapped, is plotted as dotted line, while the first and second harmonics $(l=2,3)$, which are leaky modes, are plotted as solid and dashed lines, respectively. The parameters used are $\rho_{\mathrm{l}} / \rho_{\mathrm{c}}=10$ and $b / L=0.015$. Panels c) and d) are zooms of panels a) and b) respectively to better show the behaviour in the loop region. In each panel the loop limit is marked with a long-dashed vertical line.

Table 1. Frequency, period and damping time for the first even modes with $\kappa=\pi /(2 L)(n=0)$. The equilibrium parameters are $b / L=0.015$, $\rho_{\mathrm{l}} / \rho_{\mathrm{c}}=10, L=65 \mathrm{Mm}, n_{\mathrm{p}}=5 \times 10^{14} \mathrm{~m}^{-3}$ and $B_{0}=10 \mathrm{G}$, giving an Alfvén speed of $c_{\mathrm{Ac}}=1252 \mathrm{~km} \mathrm{~s}^{-1}$.

\begin{tabular}{lc|cccc}
\hline \hline$m$ & $l$ & $\omega_{\mathrm{r}} L / c_{\mathrm{Ac}}$ & $\omega_{\mathrm{i}} L / c_{\mathrm{Ac}}$ & $T(\min )$ & $\tau(\min )$ \\
\hline 1 (kink) & 0 & 0.670 & 0 & 8.12 & $\infty$ \\
2 (fluting) & 0 & 0.670 & 0 & 8.12 & $\infty$ \\
3 (fluting) & 0 & 0.670 & 0 & 8.12 & $\infty$ \\
0 (sausage) & 0 & 48.04 & -4.98 & 0.113 & 0.17 \\
1 (kink) & 1 & 76.62 & -6.07 & 0.071 & 0.14 \\
2 (fluting) & 1 & 101.27 & -3.85 & 0.054 & 0.23 \\
3 (fluting) & 1 & 128.0 & -1.28 & 0.042 & 0.68 \\
\hline
\end{tabular}

It is important to remark that the fundamental modes with $m \geq 1$ are trapped, and have a frequency $\omega_{\mathrm{r}} L / c_{\mathrm{Ac}} \approx$ $\left(c_{\mathrm{k}} / c_{\mathrm{Ac}}\right) \pi(n+1)$ and a period $T \approx 8.12 /(n+1) \mathrm{min}$; they are not damped in this model. The first mode was used in Nakariakov \& Ofman (2001) to deduce the magnetic field strength $B_{0}=13 \pm 9 \mathrm{G}$, compared to the value used in the present paper $\left(B_{0}=10 \mathrm{G}\right)$.

\section{Effects of a chromospheric layer on the trapped modes}

In previous sections it has been made clear that for the homogeneous flux tube of Fig. 1 the wavenumber in the $z$-direction, $\kappa$, is quantized (Eq. (11)) and that for each family of solutions sharing the same $n$, a different cut-off frequency is found. However, Díaz et al. (2002) pointed out that the addition of structure along the magnetic field modifies this result, since modes with the same symmetry become mixed and the cutoff is just the lowest one $\left(\omega_{\text {cut }} L / c_{\mathrm{Ac}}=\pi / 2\right.$ for even modes, $\omega_{\text {cut }} L / c_{\text {Ac }}=\pi$ for the odd ones).

The equilibrium structure and properties of coronal loops are still being investigated, but it is clear that there is some inhomogeneity along the loop: the chromospheric layer near the photosphere (see Fig. 6 for the simplified model used here). The coronal parameters used in the previous section are again used here $\left(2 L=130 \mathrm{Mm}, 2 b=1 \mathrm{Mm}\right.$ and $\left.\rho_{\mathrm{l}} / \rho_{\mathrm{c}}=10\right)$; for the chromospheric layer a typical height $H_{\mathrm{ch}}=5 \mathrm{Mm}$ and density ratio $\rho_{\mathrm{ch}} / \rho_{\mathrm{c}}=200$ are used. Therefore, we next study how the modes described in Sect. 3 are modified when this structure is taken into account. The problem can be solved using the techniques developed in Díaz et al. (2002). Separation of variables is used to find solutions to Eqs. (1) and (2), but now 

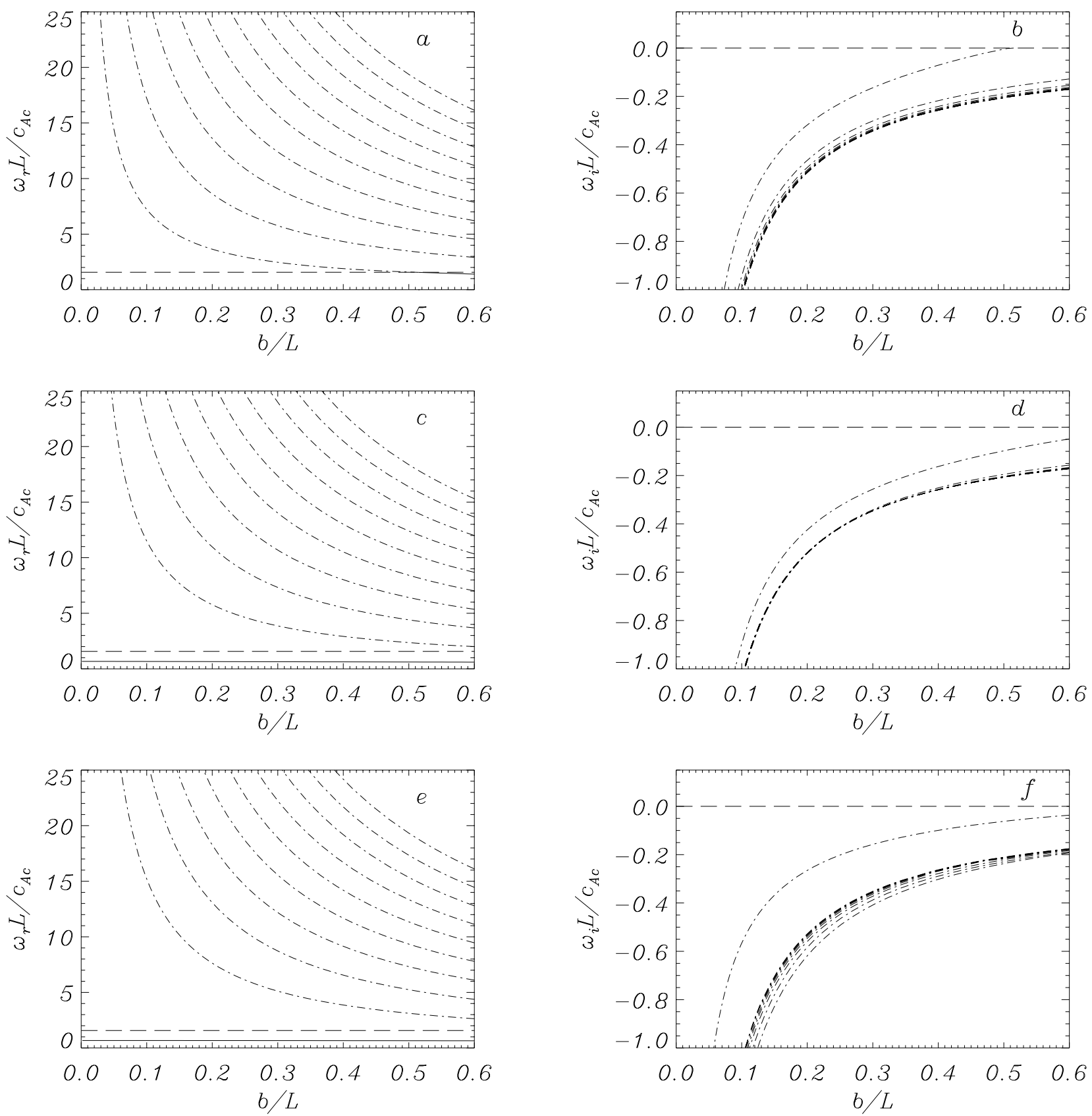

Fig. 5. Variation of $\mathbf{a}), \mathbf{c})$ and $\mathbf{e})$ the real part and $\mathbf{b}), \mathbf{d}$ ) and $\mathbf{f}$ ) the imaginary part of the dimensionless frequency with the tube radius for $n=0$ even modes. The flux tube to coronal density ratio is $\rho_{\mathrm{l}} / \rho_{\mathrm{c}}=10$. The cut-off frequency is marked with a horizontal long dashed line. Panels a) and b) correspond to the sausage modes, panels c) and d) to the kink ones and panels e) and f) to the first fluting ones $(m=2)$. In the plots of the real part a solid line denotes a trapped mode (see Fig. 3), while in the plots of the imaginary part only the values different from zero are plotted. Notice that when a mode becomes leaky (i.e. when its $\omega_{\mathrm{r}}$ surpasses the cut-off frequency) the imaginary part of its frequency is shown in the corresponding panel to the right.

the solutions cannot have a simple $z$-dependence like that in Eq. (3) and must consist instead of a sum of all the possible basis functions $h(z)$. Moreover, to match the solutions at $x=b$ the Sturm-Liouville theorem must be used to expand the inner basis functions in terms of the outer ones, leading to a system of algebraic equations for the amplitude coefficients, which constitutes the dispersion relation.

The resulting dispersion frequencies of trapped modes are plotted in Fig. 7 as a function of the loop radius. For a typical value $b / L=0.015$, sausage modes are leaky (this is true even for much larger values of $b / L$ ). In addition, there are only two trapped kink and two trapped fluting ( $m=2$, but also $m>2$, not shown here) modes, which correspond to the waves of the homogeneous tube (Sect. 3 with $n=0$ and $n=1$ and frequencies $\left.\omega_{\mathrm{r}} L / c_{\mathrm{Ac}} \approx\left(c_{\mathrm{k}} / c_{\mathrm{Ac}}\right)(n+1) \pi / 2\right)$. The other $m=1$ and $m=2$ modes with $n \geq 2$ are above the new common cut-off frequency, $\omega_{\text {cut }} L / c_{\mathrm{Ac}}=\pi / 2$, so they all are leaky. However, due to the chromospheric structure the two remaining trapped modes do not correspond to a single $n$ but have contributions from the basis functions for all values of $n$. The frequencies and 


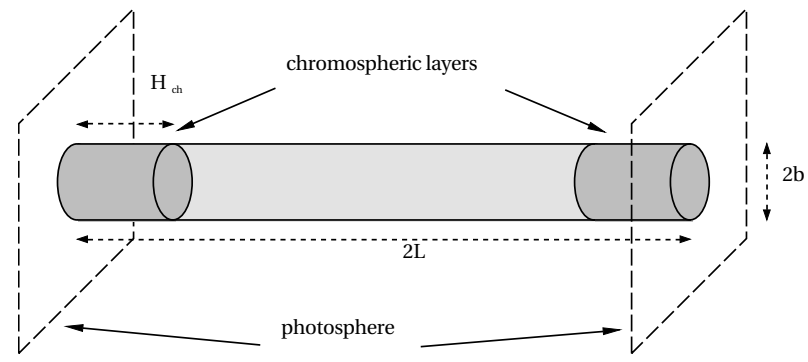

Fig. 6. Sketch of the equilibrium configuration representing a coronal flux tube of length $2 L$ and radius $b$ with chromospheric material at its footpoints (dark grey in the figure) extending up to a height $H_{\mathrm{ch}}$. The density inside the loop is $\rho_{\mathrm{l}}, \rho_{\mathrm{ch}}$ is the density in the chromospheric part and $\rho_{\mathrm{c}}$ in the coronal environment.

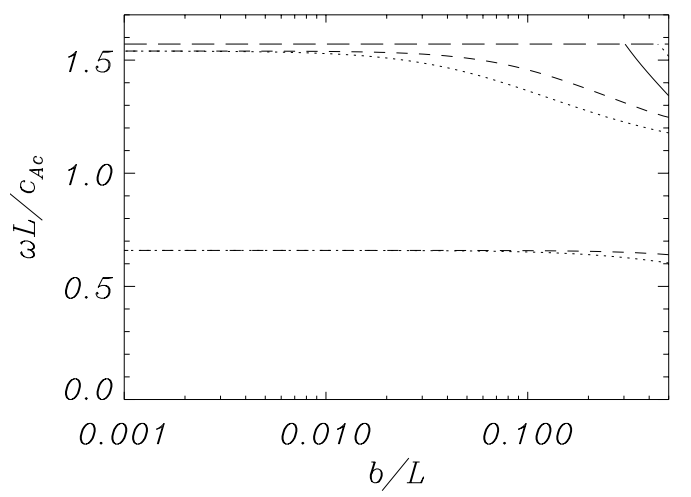

Fig. 7. Variation of the dimensionless frequency with the loop radius for even modes of a tube with density ratio $\rho_{\mathrm{l}} / \rho_{\mathrm{c}}=10$ and chromospheric density ratio $\rho_{\mathrm{ch}} / \rho_{\mathrm{c}}=200$. The solid line corresponds to the trapped sausage mode $(m=0)$, the dotted line to the kink modes $(m=1)$ and the dashed line to the first fluting modes $(m=2)$. The cut-off frequency, marked with a horizontal long dashed line, is $\omega_{\text {cut }} L / c_{\mathrm{Ac}}=\pi / 2$.

periods for the kink modes are summarized in Table 2 for the same coronal density and magnetic field strength considered in Sect. 4 . The periods of the $n=0$ and $n=1$ kink modes are only slightly modified by the presence of the chromospheric layers, so the most important result is that the higher order modes become leaky (though we have not determined their frequencies and damping times). As noted in Sect. 4, these damping times can be quite small, perhaps preventing these modes from being detected, whereas for the homogeneous tube they are non-leaky and thus detectable in principle.

The dependence of the modes on the two new parameters, $H_{\mathrm{ch}} / L$ and $\rho_{\mathrm{ch}} / \rho_{\mathrm{c}}$, is represented in Fig. 8. Notice that the frequency of the fundamental mode is almost insensitive to these parameters, but the harmonics strongly depend on them (and can even become leaky for some values).

Table 2 shows and important difference between the period of the homogeneous loop and the loop with a chromospheric layer, in particular for the $n=1$ kink mode. A possible origin for this difference could be that the dense chromospheric material does not oscillate appreciably, so that the loop has an "effective" length $2\left(L-H_{\text {ch }}\right)$ instead of $2 L$. The frequency of a homogeneous tube with this length is also represented in Fig. $8 \mathrm{~b}$ by a dashed line. The discrepancy between the solid
Table 2. Dimensionless frequency and period $T$ for the trapped kink even modes in a coronal flux tube with chromospheric layers. The parameters are $\rho_{\mathrm{l}} / \rho_{\mathrm{c}}=10, \rho_{\mathrm{ch}} / \rho_{\mathrm{c}}=200, H_{\mathrm{ch}} / L=0.1, n_{\mathrm{p}}=5 \times 10^{14} \mathrm{~m}^{-3}$ and $B_{0}=10 \mathrm{G}$. The last column gives the period of the corresponding mode for the homogeneous tube. Short period modes with $n \geq 2$ are leaky in the chromospheric model (but not in the absence of chromosphere).

\begin{tabular}{cccc}
\hline \hline$n$ & $\omega L / c_{\text {Ac }}$ & $T(\min )$ & $T_{\text {homog }}(\min )$ \\
\hline 0 & 0.659 & 8.25 & 8.12 \\
1 & 1.519 & 3.58 & 2.71 \\
2 & - & - & 1.62 \\
3 & - & - & 1.16 \\
\hline
\end{tabular}

and dashed lines in this figure proves that the shift in the period is not caused by a reduction in the "effective" length of field lines, but by the modification in the spatial profile of perturbations induced by the chromospheric layer.

\section{Conclusions}

The oscillations of coronal flux tubes with line-tying boundary conditions at the photospheric footpoints have been studied for a low beta plasma. A rich array of modes, trapped and leaky, have been classified, focusing on their frequency and spatial structure. We have also made some comparisons of our results with current observational data.

The line-tying boundary conditions introduce some important differences from the classical results for unlimited flux tubes (Edwin \& Roberts 1983):

1. The introduction of line-tying adds a new characteristic length and a quantization $(n)$ in the direction along the flux tube. The plots of the dispersion relation display these features a well as the quantizations in the azimuthal $(m)$ and radial $(l)$ directions.

2. In a straight and uniform magnetic cylinder, for values of length, thickness and density of the flux tube in accordance with observations, sausage modes $(m=0)$ are leaky since their frequency lies above the cut-off frequency. If a sausage mode is excited in the loop, its energy is quickly transferred to the coronal environment and the oscillation dies out. For all other modes $(m>0)$ at least the fundamental mode lies below the cut-off frequency. Hence, if any of these modes is excited the oscillatory energy in the loop plasma does not decay. Moreover, as the density ratio between the loop and the coronal medium $\left(\rho_{1} / \rho_{\mathrm{c}}\right)$ is increased, the frequency of other harmonics may move below the cut-off and so a dense loop may support a higher number of oscillatory modes.

3. Leaky modes have very short periods and short damping times. If these modes were excited, their energy would be quickly pumped out to the corona, making them difficult to detect (a high cadence would be necessary). Their strong attenuation would also lower their amplitude below the noise level in just a few oscillations.

4. The modes of a homogeneous flux tube with higher values of $n$, i.e. shorter wavelength along the loop, have 

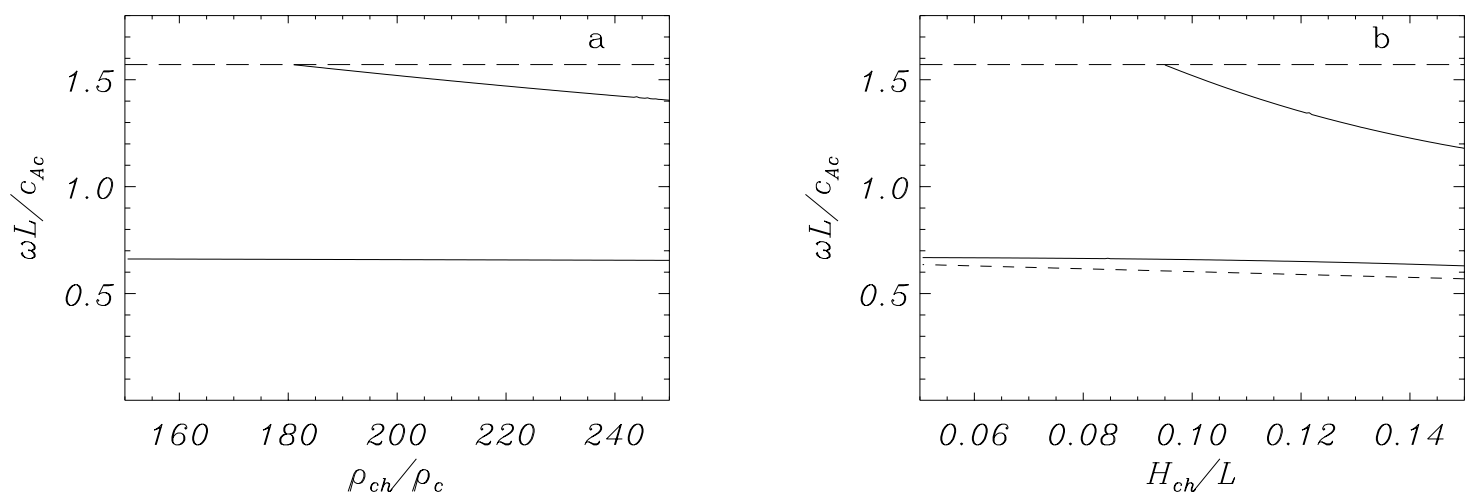

Fig. 8. Variation of the dimensionless frequency with a) the chromospheric density (and $H_{\mathrm{ch}} / L=0.1$ ) and b) the chromospheric height (and $\left.\rho_{\mathrm{ch}} / \rho_{\mathrm{c}}=200\right)$ for kink even modes of a tube with density ratio $\rho_{\mathrm{l}} / \rho_{\mathrm{c}}=10$ and radius $b / L=0.015$. The cut-off frequency, marked with a horizontal long dashed line, is $\omega_{\text {cut }} L / c_{\mathrm{Ac}}=\pi / 2$. The dashed line in panel b) represents the frequency of the only non-leaky mode of a homogeneous loop with half-length $L-H_{\mathrm{ch}}$.

periods $T=T_{0} /(n+1)$, where $T_{0}$ is the period of the mode with the same numbers $l$ and $m$, but with $n=0$. These modes could be detected, since their period is still in the range of the fundamental one and current spatial resolution is high enough. Observational data should contain signatures of these higher harmonics.

5. Currently available observational data does not usually show such short period modes. The suggested explanation is that if some structure is added to the model (for example, the chromospheric layers), the modes having the same $m$ and $l$ are combined to satisfy the MHD boundary conditions on the loop-corona interface. Under such conditions, all the higher order modes become leaky and therefore undetectable in current observations.

6. Typical calculated periods are in the range of the observational data, so we are encouraged to develop more complicated models. The results can be applied to calculate various equilibrium parameters from observations of the oscillatory properties, but the model needs to be refined first. The obvious next step would be to use a more accurate equilibrium profile than that with a simple chromospheric layer. This work is a first step towards such studies.

In summary, it has been shown that in a straight and homogeneous cylindrical flux tube there is a rich spectrum of MHD fast modes and there should be observational signatures of the excitation of higher order harmonics. Here we have also considered the addition of the chromospheric structure at the base of the loop and our results are that these modes become leaky. Leakage can be quite efficient in damping the oscillations for many of these high frequency (compared to fundamental) modes, but the detailed study of this effect is left for further work.

Additionally, we are fully aware of the number of assumptions and simplifications that have been made. As we pointed out in the introduction, there are a number of effects, such as non-homogeneities, curvature or dropping the zero-beta plasma assumption, that may have a relevant role in the oscillations of coronal loops, but we regard our results as a first step towards a better understanding of the complex array of modes supported by these structures.
Acknowledgements. A. J. Díaz, R. Oliver and J. L. Ballester acknowledge the financial support received from MCyT under grant AYA2003-00123. Financial support from NATO grant PST.CLG976135 is also acknowledged. A. J. Díaz thanks the Spanish government for a grant and the School of Mathematics and Statistics of the University of St. Andrews, in which part of this work was developed, for their kind hospitality.

\section{References}

Abramowitz, M., \& Stegun, I. A. 1967, Handbook of Mathematical Functions, Dover

Aschwanden, M. J., Fletcher, L., Schrijver, C. J., \& Alexander, D. 1999, АpJ, 520, 880

Aschwanden, M. J. 2002, in Proc. of the Magnetic Coupling of the Solar Atmosphere Euroconference and IAU Coll., 188 (ESA publications division), SP-505, 191

Aschwanden, M. J., \& Schrijver, C. J. 2002, ApJS, 142, 269

Aschwanden, M. J., De Pontieu, B., Schrijver, C. J., \& Title, A. M. 2002, Sol. Phys., 206, 99

Bennett, K., Roberts, B., \& Narain, U. 1999, Sol. Phys., 185, 41

Cally, P. S. 1986, Sol. Phys., 103, 277

Cally, P. S. 2003, Sol. Phys., 217, 95

De Pontieu, B., Martens, P. C. H., \& Hudson, H. S. 2001, ApJ, 520, 580

Díaz, A. J., Oliver, R., Edérly, R., \& Ballester, J. L. 2001, A\&A, 379, 1083

Díaz, A. J., Oliver, R., \& Ballester, J. L. 2002, ApJ, 580, 550

Díaz, A. J., Oliver, R., \& Ballester, J. L. 2003, A\&A, 402, 781

Edwin, P. M., \& Roberts, B. 1982, Sol. Phys., 76, 239

Edwin, P. M., \& Roberts, B. 1983, Sol. Phys., 88, 179

Goedbloed, J. P. 1983, Lecture Notes on Ideal Magnetohydrodynamics, Rijnhuizen Rep., 76

Goossens, M., Andries, J., \& Aschwanden, M. J. 2002, A\&A, 394, L39

Heyvaerts, J., \& Priest, E. R. 1983, A\&A, 117, 220

Hollweg, J. V. 1984, ApJ, 277, 392

Homem, M. T., Erdélyi, R., \& Ballai, I. 2003, Sol. Phys., 217, 199

Hollweg, J. V., \& Roberts, B. 1981, ApJ, 250, 398

Joarder, P. S., \& Roberts, B. 1992, A\&A, 256, 264

Karami, K., Nasiri, S., \& Sobouti, Y. 2002, A\&A, 396, 993

Katsiyannis, A. C., Williams, D. R., McAteer, R. T. J., et al. 2003, A\&A, 406, 709

Nakariakov, V. M., \& Roberts, B. 1995, Sol. Phys., 159, 213 
Nakariakov, V. M., \& Ofman, L. 2001, A\&A, 372, 53

Nakariakov, V. M., Ofman, L. Deluca, E. E., Roberts, B., \& Davila, J. M. 1999, Science, 285, 862

Ofman, L., Davila, J. M., \& Steinolfson, R. S. 1994, ApJ, 421, 360

Ofman, L., Davila, J. M., \& Steinolfson, R. S. 1994, ApJ, 444, 471

Ofman, L. 2002, ApJ, 568, L135

Ofman, L., \& Wang, T. 2002, ApJ, 580, L850

Oliver, R., Ballester, J. L., Hood, A. W., \& Priest, E. R. 1992, ApJ, 400, 369

Poedts, S., \& Goossens, M. 1991, Sol. Phys., 133, 281

Priest, E. R., Foley, C. R., Heyvaerts, J., et al. 2000, ApJ, 539, 1002

Roberts, B., Edwin, P. M., \& Benz, A. O. 1984, ApJ, 279, 857

Roberts, B. 1991, in Advances in Solar System Magnetohydrodynamics, ed. E. R. Priest, \& A. W. Hood (Cambridge Univ. Press), 110

Roberts, B. 2000, Sol. Phys., 193, 139

Roberts, B. 2002, in Proc. of the 10th European Solar Physics Meet. (ESA publications division), SP-506, 481
Ruderman, M. S., \& Roberts, B. 2002, ApJ, 577, 475

Sakai, J. I., Yawata, T., Yoshida, K., Furusawa, K., \& Cramer, N. F. 2000, ApJ, 537, 1063

Schrijver, C. J., \& Brown, D. S. 2000, ApJ, 537, L69

Schrijver, C. J., Aschwanden, M. J., \& Title, A. M. 2002, Sol. Phys., 206, 69

Smith, J. M., Roberts, B., \& Oliver, R. 1997, A\&A, 317, 752

Somasundaram, K., Venkatraman, S., \& Sengottuvel, M. P. 1999, Plasma Phys. Control Fusion, 41, 1421

Steinolfson, R. S., \& Davila, J. M. 1993, ApJ, 415, 354

Stenuit, H., Keppens, R., \& Goossens, M. 1998, A\&A, 331, 392

Testa, P., Peres, G., \& Reale, F. 2002, ApJ, 580, 1159

Wang, T. J., Solanki, S. K., Curdt, W., Innes, D. E., \& Dammasch, I. E. 2002, ApJ, 574, 101

Wang, T. J., Solanki, S. K., Innes, D. E., Curdt, W., \& Marsch, E. 2003, A\&A, 402, 17

Wang, T. J., Solanki, S. K., Curdt, W., et al. 2003, A\&A, 406, 1105 


\section{Online Material}




\section{Appendix A: Line-tied Cartesian slab}

Coronal loops are more appropriately modelled as cylindrical flux tubes than as slabs, but it is nonetheless useful to have some results in Cartesian geometry to compare with the cylindrical ones. Moreover, magnetic slabs are commonly used to model fibril structures in prominences (see, for example, Joarder \& Roberts 1992; Oliver et al. 1992; Díaz et al. 2001). Here we briefly develop expressions for slabs similar to those obtained for cylindrical geometry in the main part of this paper. The equilibrium model is similar (homogeneous dense region, uniform magnetic field parallel to the $z$-axis), but with no structure in the $y$-direction. We consider perturbations with no dependence on the $y$-coordinate, although the results can easily be extended to allow dependence on this coordinate (see Díaz et al. 2003).

The starting point is Eq. (1) for the total pressure. Separation of variables in the form

$p_{\mathrm{T}}(x, z)=u(x) h(z)$

with $h(z)$ as for cylindrical geometry (Eq. (7)), leads to

$u(x)=\left\{\begin{array}{l}A \cosh \lambda^{(1)} x, 0 \leq x \leq b, \\ B \mathrm{e}^{-\lambda^{(\mathrm{c})}(x-b)}, \quad x>b .\end{array}\right.$

Here we have taken an even solution in the $x$-direction; a similar form can be written for the odd modes by substituting the cosh function by a sinh function. Using the jump conditions of Eq. (13), the slab dispersion relations are

$\lambda^{(1)} \operatorname{coth} \lambda^{(1)} b+\lambda^{(\mathrm{c})}=0$

for the sausage modes (perturbed velocity odd with respect to $x=0)$ and

$\lambda^{(1)} \tanh \lambda^{(1)} b+\lambda^{(\mathrm{c})}=0$

for the kink modes (perturbed velocity even with respect to $x=0$ ). These dispersion relations have been widely discussed for unbounded slabs (see, for example, Edwin \& Roberts 1982).

The next feature to be studied is the thin slab limit $(b / L \rightarrow 0)$. Since $\tanh z \sim z$ for $z \ll 1$, Eq. (A.3) for the sausage modes yields

$\frac{\lambda^{(1)}}{\lambda^{(1)} b}+\lambda^{(\mathrm{c})}=0$

This equation cannot be satisfied for any value of the frequency, since $\lambda^{(\mathrm{c})}$ should go to infinity and there is no finite value of $\omega$ in Eq. (8) that can satisfy that condition; thus, the sausage mode cannot propagate in very thin slabs. For the kink modes, Eq. (A.4) in the limit $b / L \rightarrow 0$ gives

$\lambda^{(1)}\left(\lambda^{(1)} b\right)+\lambda^{(\mathrm{c})}=0$,

requiring $\lambda^{(\mathrm{c})}=0$. From Eqs. (8) and (11) this leads to

$\frac{\omega}{c_{\mathrm{Ac}}} L=\kappa L=\frac{(n+1) \pi}{2 L}, n=0,1, \ldots$

It is important to notice that there are non-vanishing amplitudes far away from the slab, since the exponential decrease in Eq. (A.2) is very slow when $\lambda^{(\mathrm{c})} \rightarrow 0$, in contrast with the cylindrical tube for which the perturbation is always confined around the density inhomogeneity (see Eq. (21)).

Our next aim is to plot the dispersion relation, Eq. (A.3). However, $\lambda^{(\mathrm{c})}$ has to be real to have trapped modes, so there are the same cut-off frequencies for each value of $\kappa$ as in a cylindrical geometry (Eq. (23)). The dispersion relations are plotted in Fig. A. 1 for modes with $n=0,1$ and 2 in a very dense slab. The reason for selecting such a high density ratio is that this kind of model applies much better to prominences as a whole than to coronal tubes. Each family has its own cut-off frequency, $\omega_{\text {cut }}$, given by Eq. (23), and in the limit $b / L \rightarrow 0$ only one mode is trapped for each value of $n$, with a frequency tending to $\omega_{\text {cut }}$; see Fig. A.1b. Moreover, the perturbations of the surviving trapped modes in the thin slab limit reach large distances from the slab, so they can hardly be considered trapped solutions! These figures are quite similar to their cylindrical counterparts (Fig. 2) for large values of the tube radius, but lower values they differ markedly. Figure A.1 can be compared with Fig. 4 of Edwin \& Roberts (1982) (corrected in Edwin \& Roberts 1983) taking into account that their plot is drawn using $\omega / \kappa$ as the dependent variable, while here $\omega L / c_{\mathrm{Ac}}$ is used, so each family of solutions has a different vertical (and also horizontal) scale.

Having discussed the trapped modes, we can also study the leaky modes in a Cartesian slab. The results are plotted in Fig. A.2, for a high density slab. For example, for a prominence modelled as a slab (Joarder \& Roberts 1992) of length $2 L \approx$ $200000 \mathrm{~km}$ and width $2 b \approx 5000 \mathrm{~km}$ we have $\omega_{i} L / c_{\mathrm{Ac}} \approx-0.2$; for a typical coronal Alfvén speed $c_{\mathrm{Ac}} \approx 1000 \mathrm{~km} \mathrm{~s}^{-1}$, this gives a damping time $\tau=8.3 \mathrm{~min}$. In Fig. A.2b it can be appreciated that higher harmonics have almost the same damping times, although their oscillatory period is rather short $(T=$ $2 \pi / \omega_{\mathrm{r}}=1.19 \mathrm{~min}$ for $l=1$ modes and even smaller for $l>1$ modes) compared with that of the corresponding standing modes, $T=19.04 \mathrm{~min}(l=0)$. However, the fundamental mode is not damped at all. 
A. J. Díaz et al.: Line-tied coronal loop oscillations, Online Material p 3
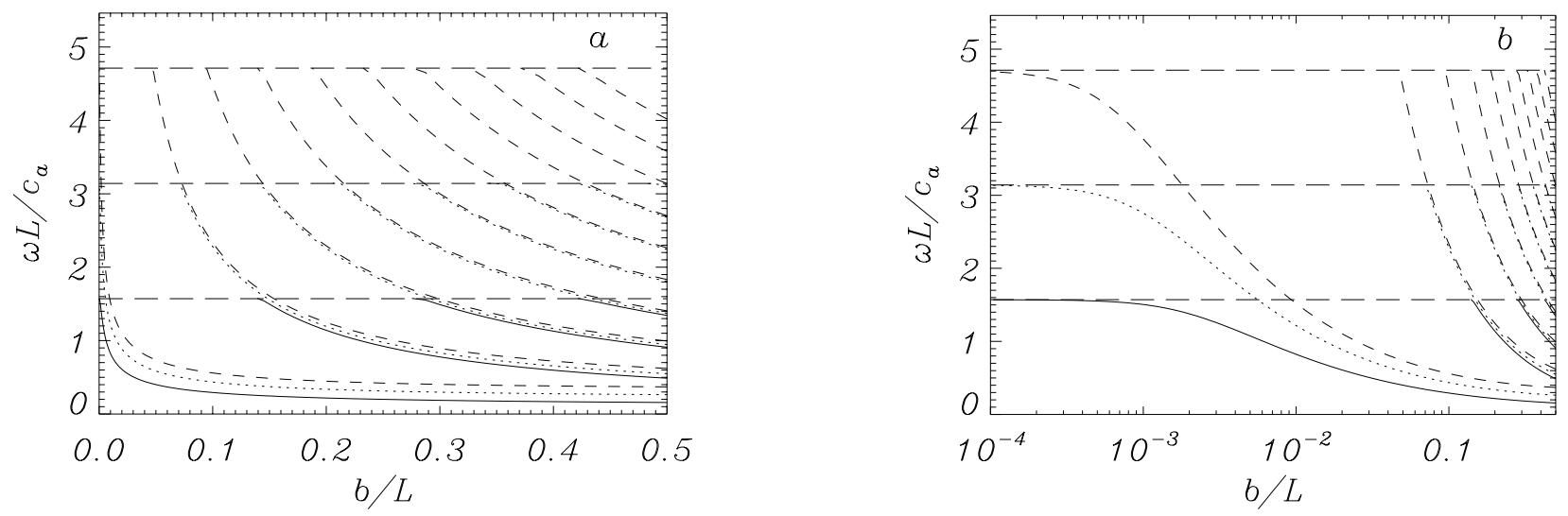

Fig. A.1. a) Variation of the dimensionless frequency with the slab half-thickness for the lowest ten kink modes of a slab with high density ratio $\rho_{1} / \rho_{\mathrm{c}}=200$ (typical of prominence plasmas). The solid lines are modes with $\kappa L=\pi / 2(n=0$, even modes), the dotted ones with $\kappa L=\pi$ ( $n=1$, odd modes) and the dashed ones with $\kappa L=3 \pi / 2(n=2$, even modes). The cut-off frequencies are marked with horizontal long dashed lines. b) Logarithmic plot of panel a), showing the structure for small $b / L$.
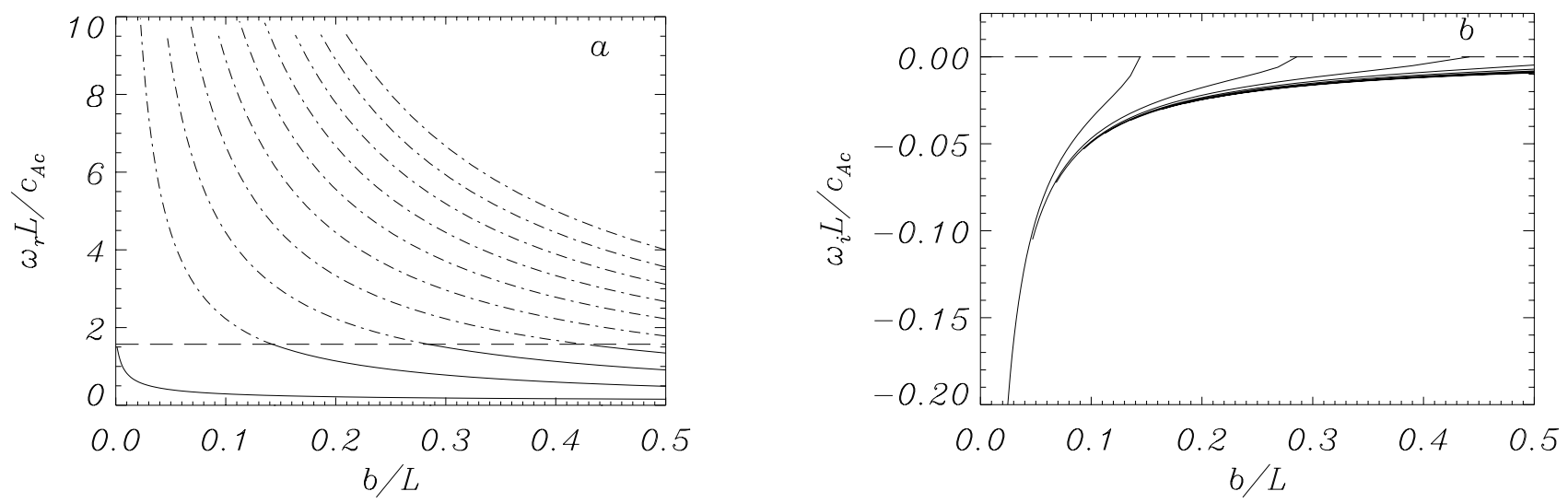

Fig. A.2. Variation of a) the real and b) the imaginary part of the dimensionless frequency with the slab half-thickness for the $n=0$ kink even modes of a slab with density ratio $\rho_{1} / \rho_{\mathrm{c}}=200$. The cut-off frequency is marked with a horizontal long dashed line. In panel a) the trapped modes are plotted with solid lines (and correspond to the solid lines in Fig. A.1), and the leaky modes with dash-dotted lines, while in panel b) only the values different from zero are plotted. Notice that when a mode becomes leaky (i.e. passes above the cut-off frequency in panel a)), its imaginary part is shown in panel $\mathbf{b}$ ). 